\title{
Low-Dissipative Hybrid Compressible Solver Designed for Large-Eddy Simulation of Supersonic Turbulent Flows
}

\author{
Yachao Lee,- - Wei Yao, $\stackrel{ \pm}{\text { and }}$ Xuejun Fan \\ Chinese Academy of Sciences, 100190 Beijing, People's Republic of China
}

\begin{abstract}
DOI: $\underline{10.2514 / 1 . J 056404}$
To reduce the numerical dissipation in turbulence modeling while maintaining the numerical stability around flow discontinuities in supersonic flowfield, a low-dissipative compressible solver is developed for large-eddy simulation within the OpenFOAM framework. To achieve the aforementioned goals, the low-dissipative solver adopts the hybrid scheme approach through combining the dissipative Kurganov-Tadmor scheme with the nondissipative central scheme via a shock sensor. In the construction of the central scheme, a robust skew-symmetric form of the convective term is adopted to preserve the local kinetic energy without adding an explicit dissipative term. Another feature of the low-dissipative solver is the implementation of an optimal explicit strong stability-preserving linear third-order total variation diminishing Runge-Kutta method for the temporal discretization. Numerical tests for a series of canonical flow problems are carried out to validate the solver's good performance in the flowfield either with strong discontinuities or with continuous spectrum characteristics. Large-eddy simulation of a scramjet combustor with supersonic airstream passing over the flame holder is conducted to validate the low-dissipative solver's reliability in a realistic flow with the complex interaction of shock discontinuities and turbulence.
\end{abstract}

\section{Nomenclature}

$C_{p} \quad=\quad$ specific heat capacity at constant pressure

$C_{v} \quad=$ specific heat capacity at constant volume

$F \quad=$ interface flux

$k \quad=\quad$ turbulent kinetic energy

$\operatorname{Pr} \quad=$ Prandtl number

$p \quad=$ pressure

$Q \quad=\quad$ second invariant of velocity gradient

$R=$ gas state constant

$T=$ temperature

$U \quad=$ velocity vector

$u, v, w=$ components of velocity vector

$V \quad=$ cell volume

$x, y, z=$ Cartesian coordinates

$\gamma=$ specific heat ratio

$\delta_{i j} \quad=$ Kronecker delta tensor

$\kappa=$ wave number/Karman constant/thermal conductivity

$\mu \quad=$ dynamic viscosity

$\rho \quad=$ density

\section{Subscripts}

$\begin{array}{ll}i, j & =\text { computational indices } \\ \mathrm{vd} & =\text { van Driest transformation } \\ w & =\text { flow property at the wall } \\ \infty & =\text { far-field conditions } \\ 0 & =\text { initial conditions }\end{array}$

Presented as Paper 2017-2444 at the 21st AIAA International Space Planes and hypersonic Technologies Conference, Xiamen, China, 6-9 March 2017; received 6 June 2017; revision received 28 February 2018; accepted for publication 13 April 2018; published online 23 May 2018. Copyright $\odot 2018$ by the American Institute of Aeronautics and Astronautics, Inc. All rights reserved. All requests for copying and permission to reprint should be submitted to CCC at www.copyright.com; employ the ISSN 0001-1452 (print) or 1533-385X (online) to initiate your request. See also AIAA Rights and Permissions www.aiaa.org/randp.

*Ph.D. Student, LHD of Institute of Mechanics; also School of Engineering Science, University of Chinese Academy of Sciences, Beijing 100049, China; liyachao@imech.ac.cn. Member AIAA.

${ }^{\dagger}$ Associate Professor, LHD of Institute of Mechanics; also School of Engineering Science, University of Chinese Academy of Sciences, Beijing 100049, China; weiyao@imech.ac.cn. Member AIAA (Corresponding Author).

${ }^{\ddagger}$ Professor, LHD of Institute of Mechanics; also School of Engineering Science, University of Chinese Academy of Sciences, Beijing 100049, China; xfan@imech.ac.cn. Lifetime Member AIAA (Corresponding Author).
Superscripts

$\begin{array}{lll}n & = & \text { time level in the time marching method } \\ + & = & \text { variables normalized by wall friction properties } \\ * & = & \text { variables normalized by the characteristic scale of the } \\ & & \text { flowfield } \\ & = & \text { fluctuations }\end{array}$

I. Introduction

A CCURATE modeling of the complex internal flow environment A in scramjet combustors poses a huge challenge to computational fluid dynamics, partially due to the coexistence of turbulence structures and complex shock-wave structures. For several decades, Reynolds-averaged Navier-Stokes (RANS) have been the routine approach for turbulence modeling. However, the unsteady interactions between turbulences and shock waves in the scramjet require a wide range of flow physics and turbulent scales to be directly resolved rather than modeled. High-resolution approaches, especially large-eddy simulation (LES), are becoming dominant in revealing the flow mixing as well as combustion physics for supersonic flows. Compared with RANS, LES raises higher requirements for the numerical methods, which are used to discretize and advance the Navier-Stokes equations. Generally, LES requires minimal numerical dissipation and dispersion over the resolved length scales. The stability and reliability of the numerical methods also need to be guaranteed, especially in supersonic flows with strong discontinuities.

The main difficulty in constructing a suitable solver for large-eddy simulation of the supersonic flow lies in the fact that it needs to treat the turbulence with broad continuous spectrum and the shock wave with strong discontinuity. To achieve these goals, a number of different strategies have been put forward, and they can be roughly classified into two categories: artificial viscosity method and hybridization formulation method. The artificial viscosity method originates from the work of Jameson et al. [1] and von Neumann and Richtmyer [2]. The main idea of this method is locally adding artificial fluid transport coefficients onto the momentum and energy equations to capture the discontinuities over a numerically resolvable scale. The hybridization formulation method is based on the idea of endowing a nondissipative baseline scheme with shock-capturing capability through local replacement with a classical shock-capturing scheme, which is made to act as a nonlinear filter [3]. Here, the nondissipative scheme, shock-capturing scheme, and an adaptive switch constitute the main features of the hybridization method. Central difference schemes are often used as the nondissipative 
baseline scheme. However, the standard central scheme tends to be unstable in high-Reynolds-number turbulence [4], and so most efforts are based on the idea of splitting the convective terms in skewsymmetric form, which implies kinetic energy preservation at the semidiscrete level [5]. As to the dissipative part, the modified StegerWarming flux vector splitting scheme [6], the piecewise parabolic method [7], advection upstream splitting method (AUSM) [8], and the more accurate essentially nonoscillatory (ENO) [9] and weighted essentially nonoscillatory (WENO) $[10,11]$ schemes are frequently used to capture the discontinuities in the hybrid method. As seen, proper specification of the adaptive switch/shock sensor is critical to the formation of a hybrid scheme, which ought to be defined so that numerical dissipation is effectively confined to shocked regions. Numerous shock sensors have been proposed, for example, the Jameson-type switch [11] based on the pressure/density field, the Ducros-type switch [12] based on the velocity/dilatation field, and the Lombardini sensor [13] based on entropy generation. Here, the Ducros-type sensor is adopted due to its simplicity and good performance in shock/turbulence interactions [14].

Although the hybrid schemes have been applied to the structured solver for many years, only some recent attempts have been made to implement it within the unstructured finite volume (FV) framework, which is more useful for realistic flow modeling with complex geometry. Peterson [6] used a fourth-order symmetric scheme to reconstruct the values at the interface and calculate the nondissipative flux and employed a modified Steger-Warming flux vector-splitting scheme to act as the shock-capturing scheme. Khalighi [15] developed a novel numerical scheme for unstructured compressible LES within the unstructured flow solver CharLES, where a secondorder ENO method was used as the shock-capturing scheme and an equivalent fourth-order central scheme served as the nondissipative scheme. Modesti and Pirozzoli [8] implemented a second-order central scheme in skew-symmetric form to evaluate the nondissipative flux and a numerical diffusion scheme inherited from AUSM to act as the shock-capturing scheme. In addition to the work focused on hybrid methods, Vuorinen et al. [16] proposed a scaleselective mixed central/upwind discretization coupled with a lowdissipative Runge-Kutta projection method for time integration into OpenFOAM, which proved to be a suitable method for the highquality large-eddy simulation.

Different from the former research, in this study, a new lowdissipative compressible solver will be constituted with a different dissipative scheme and a robust central scheme, and it will be optimized to be applicable to supersonic turbulent flows with strong shock/turbulence interactions. In this new hybridization method, an updated central-upwind scheme, named as the Kurganov-Tadmor scheme [17], is adopted as the shock-capturing scheme, and the central scheme in a robust skew-symmetric form is implemented to investigate the solver's performance in resolving turbulence in viscous flow as well as holding stability in the inviscid limit. An optimal explicit strong stability-preserving linear Runge-Kutta method for third-order temporal discretization and a hybrid RANS/ LES approach are implemented into the solver to further improve its capability in LES applications. A series of canonical flow problems with increasing geometric and flow complexity will be conducted to examine the performance of the low-dissipative solver in flows with either strong discontinuities or continuous spectrum characteristics. Finally, the compressible solver with the preceding fully validated methods will be used to simulate a supersonic airstream over the cavity in a scramjet combustor with complex shock/turbulence interactions.

\section{Numerical Methods}

In this study, the three-dimensional Navier-Stokes equation for the viscous compressible perfect-gas flows are solved:

$$
\frac{\partial \bar{\rho}}{\partial t}+\frac{\partial \bar{\rho} \tilde{u}_{i}}{\partial x_{i}}=0
$$

$$
\begin{gathered}
\frac{\partial}{\partial t}\left(\bar{\rho} \tilde{u}_{i}\right)+\frac{\partial}{\partial x_{j}}\left(\bar{\rho} \tilde{u}_{i} \tilde{u}_{j}\right)=-\frac{\partial p}{\partial x_{i}}+\frac{\partial}{\partial x_{j}}\left(\bar{t}_{i j}+\bar{\tau}_{i j}^{\mathrm{sgs}}\right) \\
\frac{\partial}{\partial t}(\bar{\rho} \tilde{E})+\frac{\partial}{\partial x_{j}}\left(\bar{\rho} \tilde{u}_{j} \tilde{E}+\tilde{u}_{j} p\right)-\frac{\partial}{\partial x_{j}}\left(\tilde{u}_{i}\left(\bar{t}_{i j}+\bar{\tau}_{i j}^{\mathrm{sgs}}\right)\right) \\
=\frac{\partial}{\partial x_{j}}\left(\kappa \frac{\partial \tilde{T}}{\partial x_{j}}+\frac{\mu_{T}^{\mathrm{sgs}} C_{p}}{P r_{T}} \frac{\partial \tilde{T}}{\partial x_{j}}+\left(\mu+\frac{\mu_{T}^{\mathrm{sgs}}}{\sigma_{k}}\right) \frac{\partial k^{\mathrm{sgs}}}{\partial x_{j}}\right) \\
p=\bar{\rho} R \tilde{T}
\end{gathered}
$$

where - and $\sim$ represent the Reynolds and Favre average, respectively; the superscript "sgs" represents the subscale term; $u_{i}$ is the velocity in the $x_{i}$ direction; $\rho$ is the density; $T$ is the temperature; $p$ is the pressure; $E$ is the total internal energy; $k^{\text {sgs }}$ is the subgrid turbulent kinematic energy; $\mu$ is the dynamic viscosity; $\mu_{T}^{\mathrm{sgs}}$ is the subgrid turbulent eddy viscosity; $\kappa$ is the thermal conductivity; $C_{p}$ is the specific heat capacity at constant pressure; and $\sigma_{k}$ and $P r_{T}$ are the model coefficients. The set of the conservation equations is closed with the constitutive relations for a Newtonian fluid, whereby the viscous stress tensor $\bar{t}_{i j}$ and subgrid stress tensor $\bar{\tau}_{i j}^{\text {sgs }}$ are prescribed as

$$
\bar{t}_{i j}=2 \mu \tilde{S}_{i j}-\frac{2}{3} \mu \tilde{S}_{k k} \delta_{i j} \quad \bar{\tau}_{i j}^{\mathrm{sgs}}=2 \mu_{T}^{\mathrm{sgs}}\left(\tilde{S}_{i j}-\frac{1}{3} \frac{\partial \tilde{u}_{i}}{\partial x_{i}} \delta_{i j}\right)-\frac{2}{3} \bar{\rho} k^{\mathrm{sgs}} \delta_{i j}
$$

where $S_{i j}$ is the strain-rate tensor. In the low-dissipative solver, a hybrid RANS/LES method is used to model the effect of unresolved part and deal with the near-wall flow, which will be briefly introduced at the end of this section.

The general formulation of a hybrid low-dissipative scheme is accomplished by the linear combination of the nondissipative central scheme and a conventional dissipative scheme through the shock sensor. In this study, the dissipative flux is calculated by using the Kurganov-Tadmor scheme. The scheme is a new central-upwind scheme developed based on the Lax-Friedrichs scheme [18] and the Nessyahu-Tadmor scheme [19], and it bears a much smaller numerical viscosity than its counterparts [17]. Within the FV method framework, the formulation of the convective terms is written as follows [20]:

$$
\begin{aligned}
\int_{V} \nabla \cdot[\boldsymbol{U} \varphi] \mathrm{d} V & =\int_{S}[\boldsymbol{U} \varphi] \cdot \mathrm{d} \boldsymbol{S} \approx \sum_{f} \boldsymbol{S}_{f} \cdot \boldsymbol{U}_{f} \varphi_{f}=\sum_{f} \psi_{f} \varphi_{f} \\
& =\sum_{f}\left[\alpha \psi_{f+} \varphi_{f+}+(1-\alpha) \psi_{f-} \varphi_{f-}+\omega_{f}\left(\varphi_{f-}-\varphi_{f+}\right)\right] \\
& =F_{d}
\end{aligned}
$$

where $\Psi_{f}$ is the volumetric flux $\boldsymbol{S}_{f} \cdot \boldsymbol{U}_{f} ; \varphi_{f}$ stands for conservative variables $\left((\rho, \rho \boldsymbol{U}, \rho E)_{f} ; \alpha\right.$ is the weighing factor; $\omega_{f}$ represents the diffusive volumetric flux; $F_{d}$ is the dissipative interference flux; and $\boldsymbol{S}_{f}$ is the normal surface vector.

As to the nondissipative part, to ensure the numerical stability of the solver, the central scheme in its skew-symmetric form is often used, which usually has the following formulation proposed by Feiereisen [21]:

$$
\frac{\partial \rho u_{i} \phi}{\partial x_{i}}=\frac{1}{2} \frac{\partial \rho u_{i} \phi}{\partial x_{i}}+\frac{1}{2} \phi \frac{\partial \rho u_{i}}{\partial x_{i}}+\frac{1}{2} \rho u_{i} \frac{\partial \phi}{\partial x_{i}}
$$

where $\phi$ stands for a generic transported scalar property, such as $\boldsymbol{U}$ for the momentum equation and $E$ for the energy equation, but unity for the continuity equation.

Although it is proved to minimize the aliasing error of the central scheme, such arrangement seems suitable for incompressible flow or the flow with weak density variations. For that reason, Kennedy and Gruber [22] put forward a more robust formulation for the flow with 
strong density variations through fully expanding the convective derivatives with the triple products:

$$
\begin{gathered}
\frac{\partial \rho u_{i} \phi}{\partial x_{i}}=\alpha \frac{\partial \rho u_{i} \phi}{\partial x_{i}}+\beta\left(\rho \frac{\partial u_{i} \phi}{\partial x_{i}}+\phi \frac{\partial \rho u_{i}}{\partial x_{i}}+u_{i} \frac{\partial \rho \phi}{\partial x_{i}}\right) \\
+(1-\alpha-2 \beta)\left(\rho u_{i} \frac{\partial \phi}{\partial x_{i}}+\rho \phi \frac{\partial u_{i}}{\partial x_{i}}+u_{i} \phi \frac{\partial \rho}{\partial x_{i}}\right)
\end{gathered}
$$

where $\alpha$ and $\beta$ are the adjustable parameters. Pirozzoli [23] found that this arrangement leads to semidiscrete energy conservation in the case $\alpha=\beta=1 / 4$. Such arrangement could be easily be transformed into the flux-based form as follows [24]:

$$
\int_{V} \nabla \cdot[\rho \boldsymbol{U} \phi] \mathrm{d} V \approx \sum_{f} \boldsymbol{S}_{f} \cdot \frac{\rho_{P}+\rho_{N}}{2} \frac{\boldsymbol{U}_{P}+\boldsymbol{U}_{N}}{2} \frac{\phi_{P}+\phi_{N}}{2}=F_{c}
$$

where subscripts $P$ and $N$ stand for the values of owner and neighbor cells, respectively, and $F_{c}$ represents the nondissipative interference flux. The relationship of the aforementioned elements in the FV method is illustrated in Fig. 1.

In the hybridization formulation, the shock sensor is used as a switch, which dynamically adjusts the solver for conditions near to and away from flow discontinuities. The Ducros sensor represents a simple and frequently adopted choice, which is capable of selectively isolating genuine shocks compared with other shock sensors. In this paper, a modified formulation of the Ducros sensor proposed by Bhagatwala and Lele [25] is adopted as follows:

$$
\begin{aligned}
\Theta= & \frac{1}{2}\left(1-\tanh \left(2.5+10 \frac{\Delta}{c} \nabla \cdot U\right)\right) \\
& \times \frac{(\nabla \cdot U)^{2}}{(\nabla \cdot U)^{2}+(\nabla \times U)^{2}+\varepsilon}
\end{aligned}
$$

where $c$ is the local speed of sound, $\epsilon$ is a small value to prevent division by zero, and $\Delta$ is the cell length scale. For the sake of numerical stability, the shock sensor in this study is bounded by 0.1 and 1.0, unless otherwise specified. It is worth noting that the vorticity field is none in the one-dimensional case, and so we have to specify a finite value to limit the dissipative part only taking effect in regions with large divergence.

In summary, the convective term in the hybrid low-dissipative solver is implemented as follows:

$$
\int_{V} \nabla \cdot[\rho \boldsymbol{U} \phi] \mathrm{d} V \approx \Theta_{f} F_{d}+\left(1-\Theta_{f}\right) F_{c}
$$

where $\Theta_{f}$ is the shock sensor on the interface. In this formulation, the dissipative scheme will take effect when the shock sensor approaches unity near the flow discontinuities, and the nondissipative scheme will be dominant when the shock sensor diminishes to zero in the continuous flow region.

The time integration of the low-dissipative solver is carried out by a third-order strong stability-preserving Runge-Kutta scheme, as described by Shu and Osher [26] and Gottlieb et al. [27,28]. Let $y^{n}$ be the value after $n$ time steps; then, the general form can be written as

$$
\begin{aligned}
y^{(1)} & =y^{n}+\Delta t L\left(y^{n}\right) \\
y^{(2)} & =\frac{3}{4} y^{n}+\frac{1}{4} y^{(1)}+\frac{1}{4} \Delta t L\left(y^{(1)}\right) \\
y^{n+1} & =\frac{1}{3} y^{n}+\frac{2}{3} y^{(2)}+\frac{2}{3} \Delta t L\left(y^{(2)}\right)
\end{aligned}
$$

Here, $y$ represents the conservative variables $\rho, \rho \boldsymbol{U}$, and $\rho E$. $\Delta t$ is the time-step size, and $L(y)$ is the spatial derivative. The residuals are updated after each substep by using the latest values of the conservative variables. The time step of an explicit method is limited by the stability criterion set by the Courant-Friedrichs-Lewy (CFL) coefficient [28], which has an optimal value of 1.0 for the currently used formula.

To better treat the near-wall boundary layer and the internal turbulent flow, a hybrid RANS/LES approach known as improved delayed-eddy simulation (IDDES) based on the $k-\omega$ shear-stress transport (SST) model is applied for the modeling of the scramjet combustor. The IDDES approach is suitable for dealing with flow separation and bears the merit of alleviating the mesh resolution requirement in the near-wall regions by using the RANS mode and the rest the LES mode. In this study, the construction of the IDDES model follows the work of Gritskevic et al. [29], and constant turbulent Prandtl number $\operatorname{Pr}_{T}=0.9$ is set to close the energy equation.

In the following section, the low-dissipative solver, which adopts the hybrid spatial discretization scheme and third-order total variation diminishing Runge-Kutta temporal discretization method, is named as hybridCentralFoam. The counterpart dissipative solver, which is still named as rhoCentralFoam, follows the same formulation but with the shock sensor set to a constant value of 1.0 to revert to the shock-capturing scheme everywhere. Both solvers will be computed and compared to validate the advantage of the new lowdissipative hybrid scheme in modeling the supersonic turbulent flows via the LES approach.

\section{Results}

\section{A. Sod Shock Tube}

The Sod shock-tube problem [30] is a common test case to examine the dissipative discontinuity-capturing schemes. The initial flow variable distributions follow the shock-tube experiment expressed by Eq. (10). The gas obeys the equation of state for calorically perfect gas with the specific heat ratio of $\gamma=1.4$ :

$$
(\rho, u, p)= \begin{cases}(1,0,10), & x \leq 0 \\ (0.125,0,1), & x>0\end{cases}
$$

The flowfield evolves from the initial conditions at $t=0$, just as the diaphragm instantaneously bursts. The initial interface soon forms a right-moving shock, a left-moving rarefaction fan, and an intermediate contact discontinuity. Figure 2 shows the computed and the analytic density (Fig. 2a) and pressure profiles (Fig. 2b) at a nondimensional time of $t=0.007$. With the current method, all waves are correctly captured at their right propagation speed. Although both the contact and the shock discontinuity are smeared over a few cells, their behaviors (e.g., location and jump) are well captured. The performance of the current shock-capturing methods in capturing one-dimensional discontinuity is satisfactory.
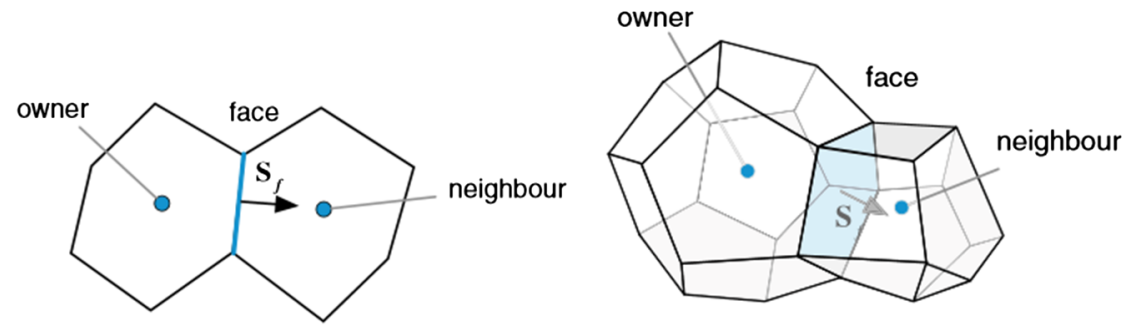

Fig. 1 Control volume for FV discretization in OpenFOAM. 


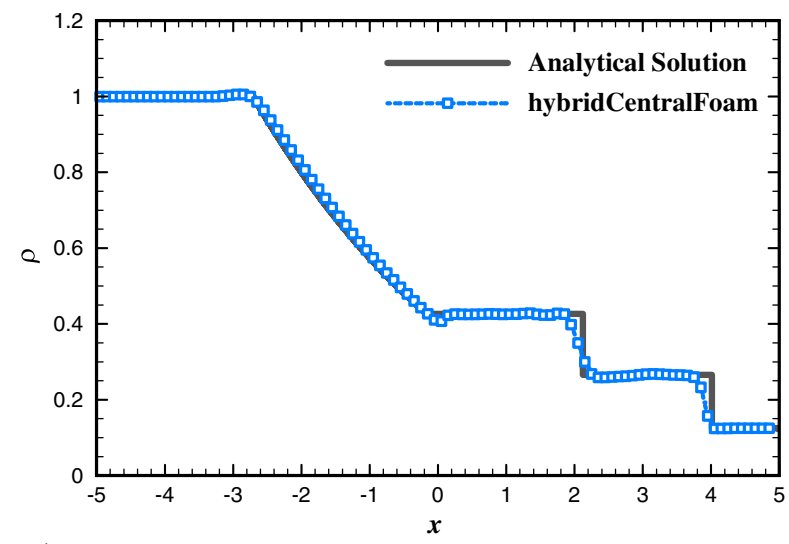

a)

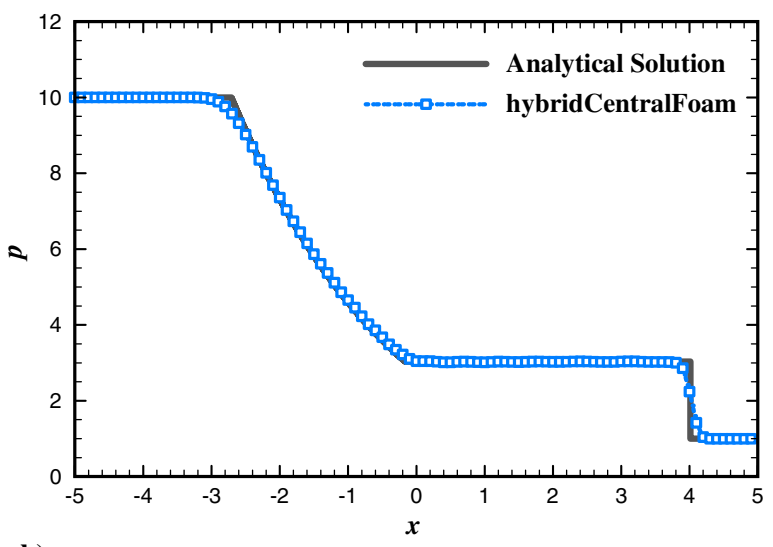

b)

Fig. 2 Profiles of a) density, and b) pressure, for the Sod shock-tube problem.
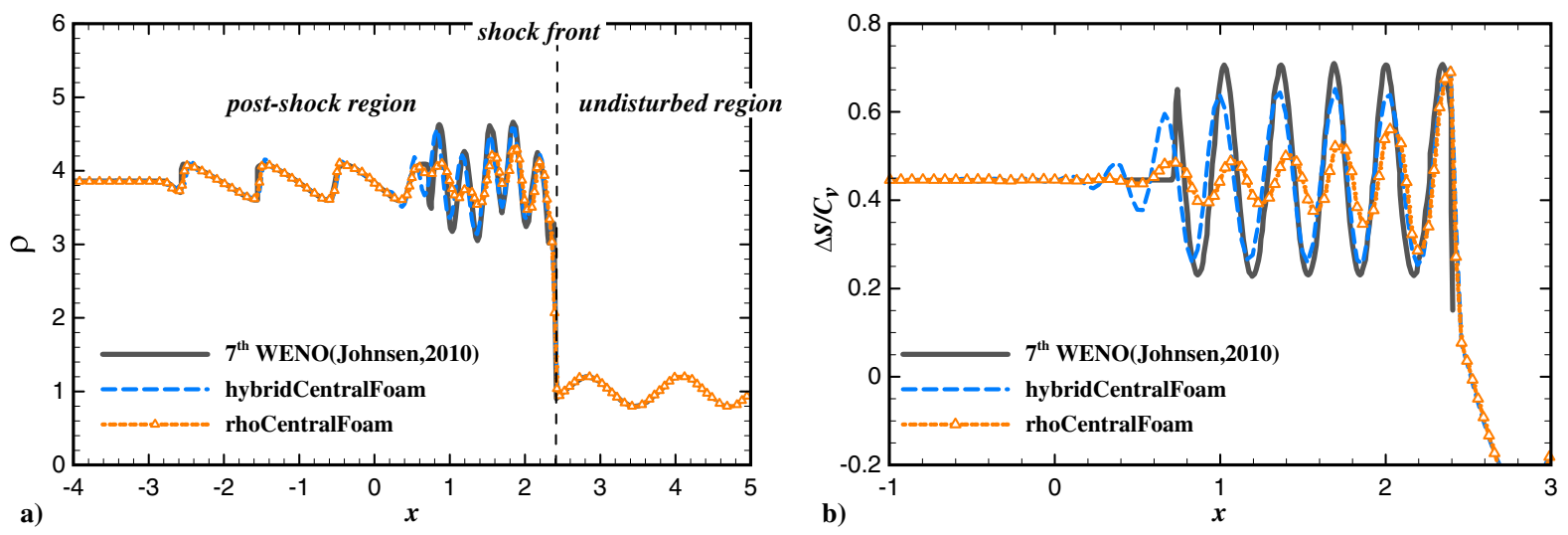

Fig. 3 Profiles of a) density, and b) entropy, for the Shu-Osher problem.

\section{B. Shu-Osher Test Case}

The Shu-Osher problem is a one-dimensional idealization of the shock-turbulence interaction problem, where a shock at $M=3$ propagates into a perturbed sinusoidal density field [31]. A complete capture of the shock discontinuity and all relevant physical wavelengths downstream of the shock requires a high-quality solver with good resolution. This makes this simple case particularly relevant to the modeling of the shock/turbulence interactions.

The case is conducted on the domain of $x \in[-5,5]$ with uniform grid spacing $\Delta x=10 / 300$. The initial conditions for this problem are given as follows:

$$
(\rho, u, p)= \begin{cases}(3.857143,2.629369,10.33333), & x<-4 \\ (1+0.2 \sin (5 x), 0,1), & x \geq-4\end{cases}
$$

Figure 3 shows the density and entropy profiles at $t=1.8$, which are compared with [32], which was obtained with 1600 points using the seventh-order-accurate WENO solution. The entropy is defined by $\Delta s / c_{v}=\ln \left(p / \rho^{\gamma}\right)$, where $c_{v}$ is the specific heat at constant volume. From Fig. 3 a, the two solvers can accurately capture the shock front and resolve the undisturbed region. However, the dissipative solver obviously smears the short-wavelength oscillations in the postshock region. In contrast, the low-dissipative solver reasonably captures most of the short wavelengths by adaptively employing the nondissipative scheme to resolve the postshock region. Similar results are found in the entropy wave field shown in Fig. 3b. For both solvers, the full entropy amplitude is accurately predicted immediately behind the shock. However, in the postshock region, the entropy wave substantially maintains its amplitude for the low-dissipative solver but decreases rapidly for the dissipative solver due to high numerical dissipation. The case demonstrates that the low-dissipative solver is not only able to accurately capture shock wave but also could properly deal with the flowfield away from the discontinuities.

\section{Taylor-Green Vortex Problem}

The Taylor-Green vortex problems are typically used to assess the discretization schemes for their scale-resolving performance. Here, both the inviscid and viscous Taylor-Green vortex problems are investigated. In the inviscid flow, the Taylor-Green vortex problem by Johnsen et al. [32] is used to examine the stability of the nondissipative scheme for severely underresolved scales and to test its ability of temporally conserving the total kinetic energy. The viscous Taylor-Green problem is used to assess the solver's performance in modeling the flowfield from a smooth initial condition to fully developed turbulence after a series of vortex

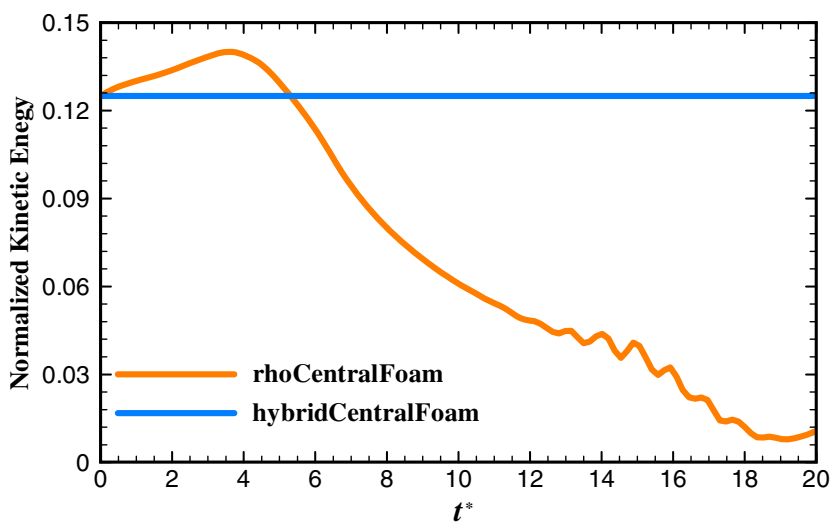

Fig. 4 Temporal evolution of total kinetic energy for the inviscid Taylor-Green vortex. 
stretching and transition. In this case, the shock-capturing scheme is disabled, and no subgrid-scale turbulence model is used.

Calculations are performed on three grid resolutions: $64^{3}, 128^{3}$, and $256^{3}$, with uniform grid spacing throughout the domain. Periodic boundary conditions are applied to all directions. The computational time $t$ is normalized by a characteristic time scale of $t^{*}=t /\left(l / U_{0}\right)$, where reference length $l=0.01 \mathrm{~m}$ and the velocity $U_{0}=$ $34.6115 \mathrm{~m} / \mathrm{s}$. All the calculations are run until 20 characteristic time scales and the maximum CFL number is no more than 0.3 .

First, numerical investigations are conducted to examine the stability of the smooth flow modeled by the nondissipative scheme when the viscous terms are removed from the Navier-Stokes equations. The temporal evolution of the total kinetic energy $E_{k}$ in the computational domain is plotted in Fig. 4, which shows that the low-dissipative solver can preserve the kinetic energy at a constant level for a long time. It should be mentioned that the total kinetic energy with the dissipative solver diverges shortly after the computation starts and then dissipates toward zero. Because there is no physical dissipation mechanism in this case, the flowfield ought to be able to recover its initial state if the time integration method is exact enough, as revealed by Duponcheel et al. [33]. Here, the sign of the velocity field is reversed at $t^{*}=10$, and the calculation is advanced until $t^{*}=20$. As shown in Fig. $\underline{5}$, the flowfield begins to evolve from the initial Green-Taylor vortex flowfield and develops into "fully turbulent" at the reversed time, and then the flowfield gradually returns to its initial state at the corresponding moment. The time reversibility of the inviscid case further confirms the kinetic energy conservation property of the skew-symmetric scheme

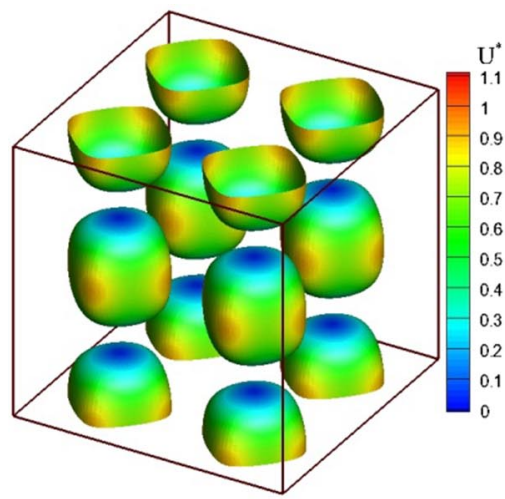

a) $\mathbf{t}^{*}=\mathbf{0}$

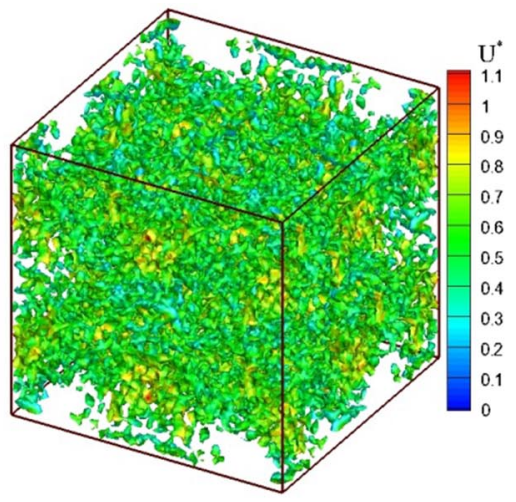

b) $\mathbf{t}^{*}=\mathbf{1 0}$

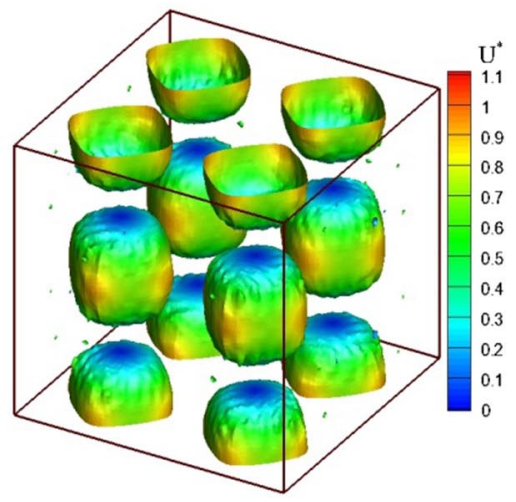

c) $\mathbf{t}^{*}=\mathbf{2 0}$

Fig. 5 Temporal evolution of the inviscid Taylor-Green vortex with time reversed at $t^{*}=10$, visualized by the isosurface of $Q$-criterion with mapped-on normalized velocity magnitude.

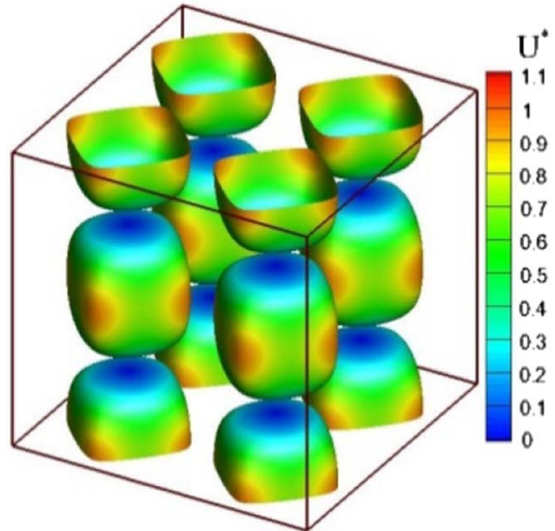

a) $t^{*}=0$

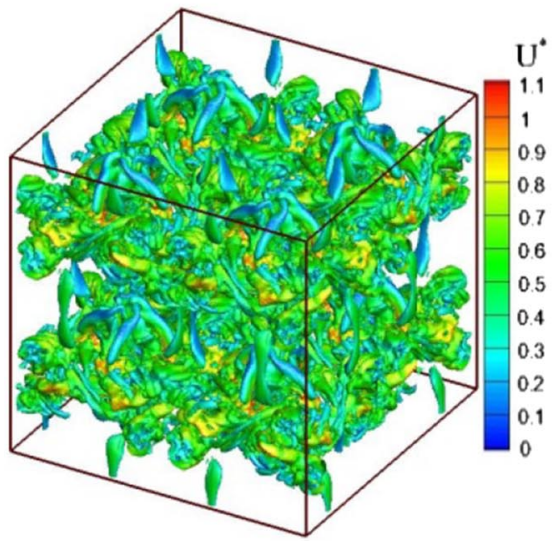

d) $\mathbf{t}^{*}=9.0$

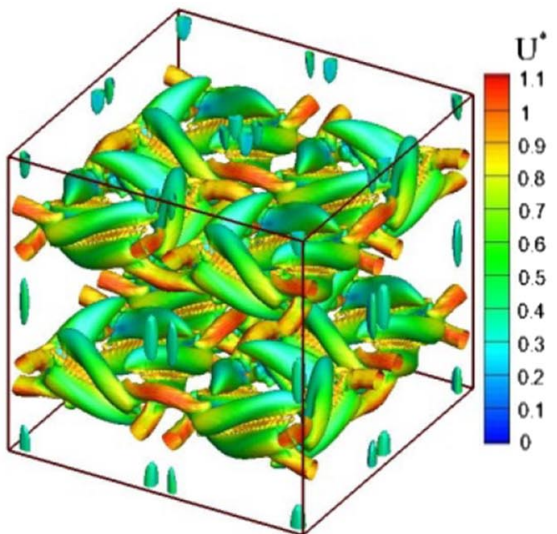

b) $\mathbf{t}^{*}=5.2$

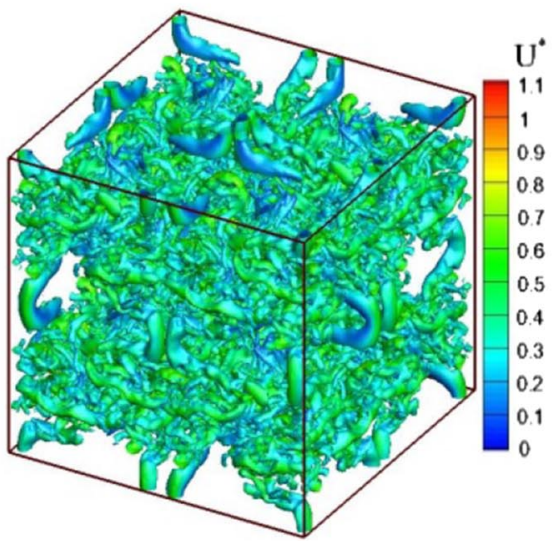

e) $t^{*}=12.1$

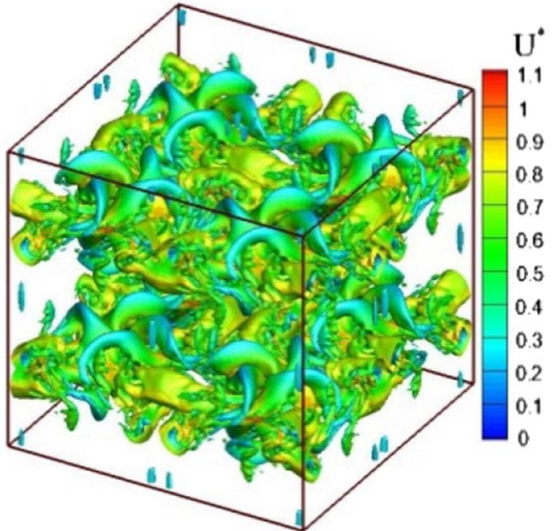

c) $t^{*}=6.9$

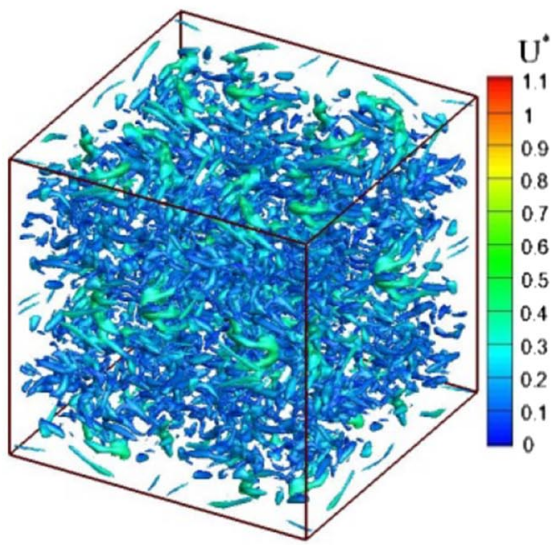

f) $\mathbf{t}^{*}=\mathbf{2 0}$

Fig. 6 Temporal evolution of the Taylor-Green vortex at $R e=1600$ visualized by isosurface of Q-criterion with mapped-on normalized velocity magnitude. 


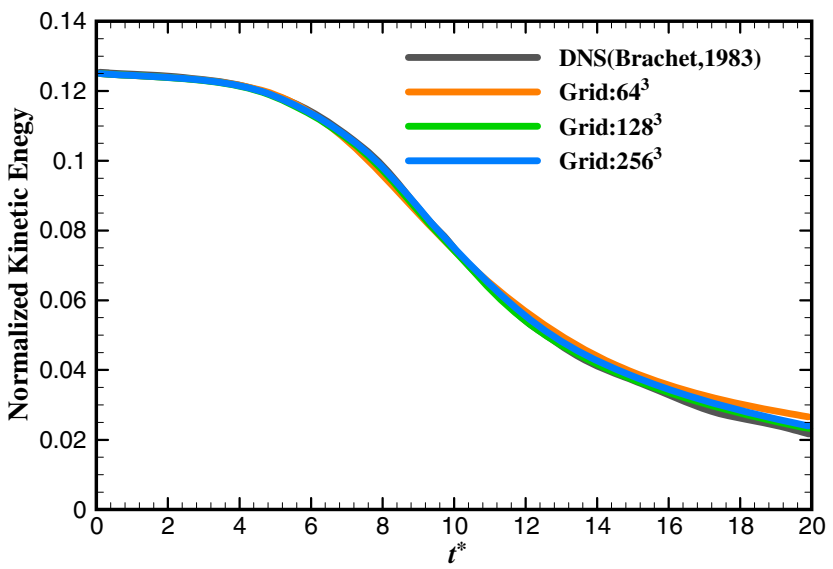

Fig. 7 Temporal evolution of total kinetic energy for the viscous TaylorGreen vortex at $R e=1600$.

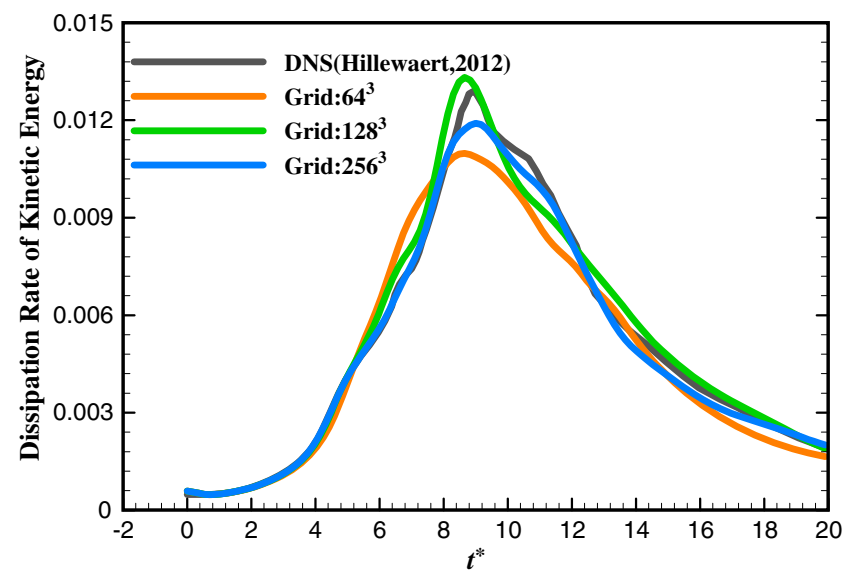

Fig. 8 Temporal evolution of the dissipation rate of total kinetic energy for the viscous Taylor-Green vortex at $R e=1600$.

implemented here, which is beneficial to the numerical stability of the low-dissipative solver.

Second, the viscous Taylor-Green vortex problem is solved with a Reynolds number of $R e=1600$ based on the reference length $l$ and the velocity $U_{0}$. No turbulence model is added, and the results will be compared with the spectral direct numerical simulation (DNS) [34,35]. The temporal evolution of the Taylor-Green vortex flowfield is visualized via the isosurface of the Q-criterion in Fig. 6. At the earliest time, the initial large-scale vortex structures begin to evolve and roll up. At around $t^{*}=6.9$, the smooth vertical structures induce more coherent structures, and at around $t^{*}=9.0$, the coherent structures begin to break down and start to transition to turbulence. From this time, the flow becomes fully turbulent and proceeds steadily to a decaying isotropic state until the flow comes to rest.

The evolutionary history of total kinetic energy $E_{k}$ for the viscous cases at different grid resolutions are plotted in Fig. 7, where the results are in good agreement with the DNS data. To further validate the performance of the skew-symmetric form in turbulence energy transfer, the temporal development of the dissipation rate of total

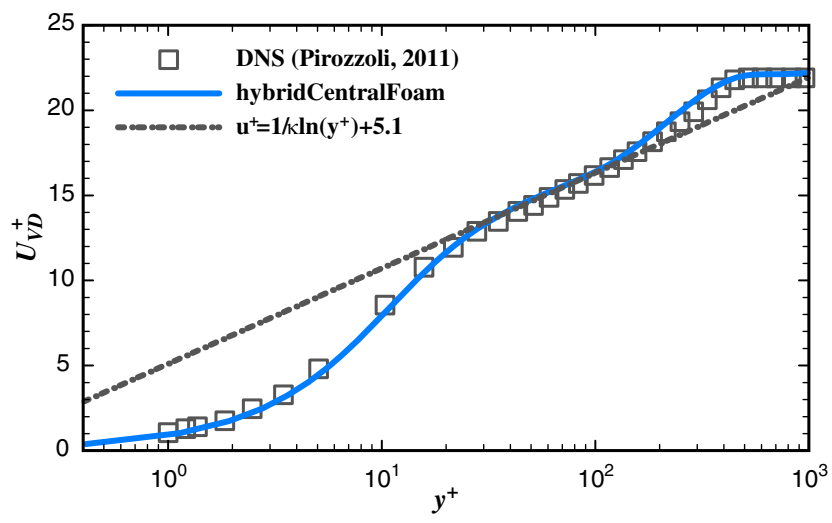

Fig. 10 Spanwise averaged mean velocity profile at location $x=6 \delta$.

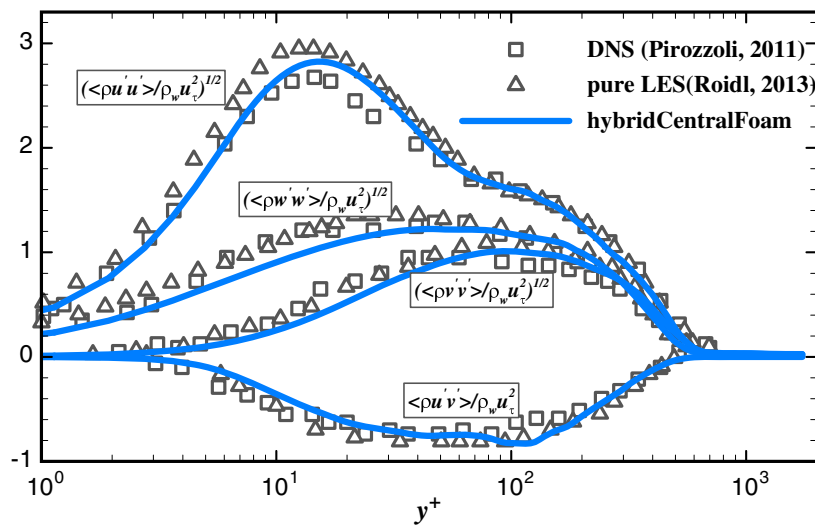

Fig. 11 Normalized streamwise, spanwise, and wall-normal rms velocity fluctuations and Reynolds shear stress profiles at location $x=6 \delta$.

kinetic energy $\varepsilon=-\mathrm{d} E_{k} / \mathrm{d} t$ is plotted in Fig. $\underline{8}$. The good agreement for all the three resolutions indicates that the energy cascades from large to small scales in such a complex flow development have been accurately predicted.

\section{Fully Developed Turbulent Flow}

To assess the capability of the low-dissipative solver in dealing with compressible wall-bounded flows in the LES framework, a supersonic crossflow over a flat plate with the freestream Mach number 2.0 is investigated. Within the boundary layer, the large vorticity will diminish the Ducros-type sensor to the threshold value, and then the central scheme will become dominant. The turbulent model is added in this case by activating the IDDES approach based on Menter's $k-\omega$ SST model.

The computational domain size for the LES calculation is $24 \delta$, $2.5 \delta$, and $5 \delta$ in streamwise, spanwise, and wall-normal directions, respectively. Uniform grid spacing, $d x^{+}=25$ and $d z^{+}=10$, is used in streamwise and spanwise directions up to $x=20 \delta$. In the wallnormal direction, the grid is clustered from $y^{+}=60$ at the upper edge to $y^{+}=1$ at the wall, and the maximum grid stretching factor is no more than 1.15. Periodic boundary conditions are applied in the spanwise direction for all variables, and a no-slip boundary condition

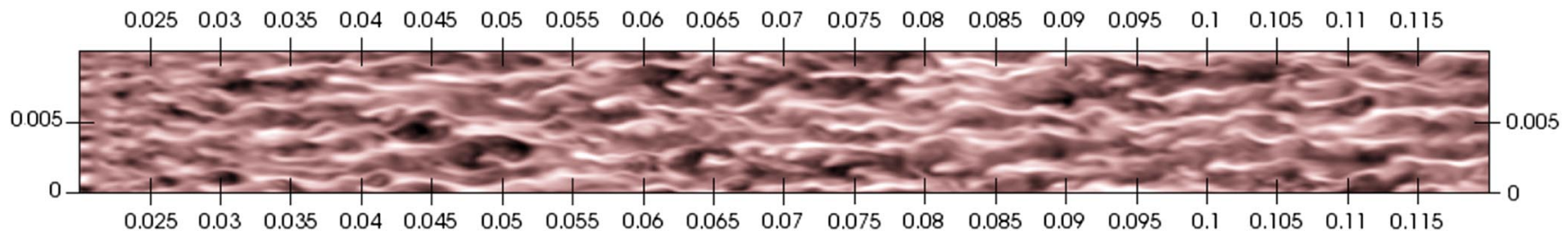

Fig. 9 Instantaneous streamwise velocity fluctuation field at a wall-normal distance $y^{+}=30$, contour levels are shown for $-0.25<U_{x}^{\prime} / U_{\infty}<0.25$, from dark to light shades. 


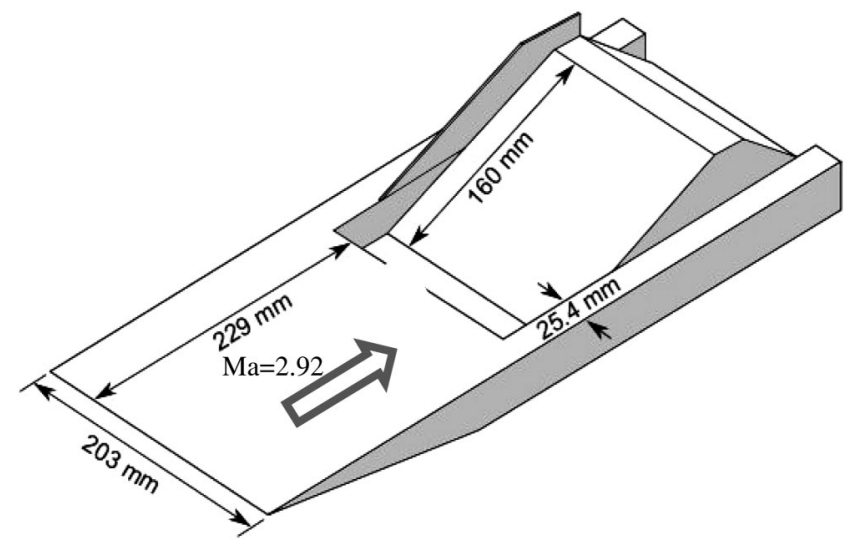

Fig. 12 Geometry sketch of the experimental model.

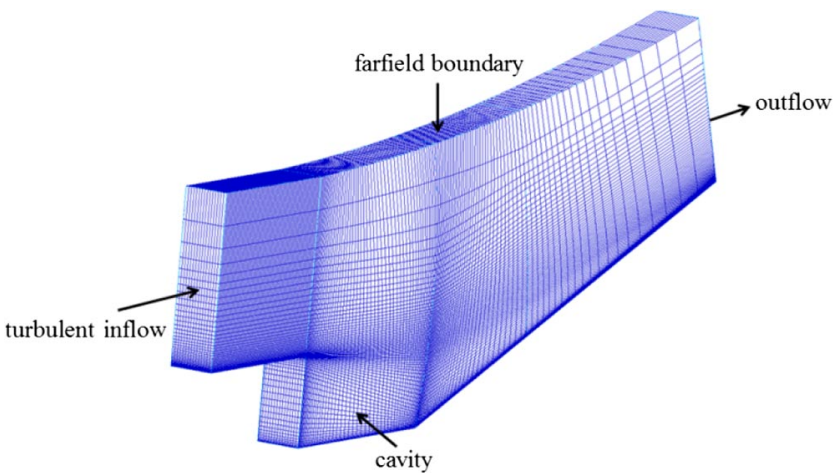

Fig. 13 Isometric view of the computational domain with coarsened grids for visual clarify.

is imposed upon the adiabatic wall. A reformulated synthetic turbulence generation method developed by Roidl et al. [36] is employed to generate a time-varying inflow boundary conditions. The time-step size in this case is set to $\Delta t=3 e-8 \mathrm{~s}$, which corresponds to a nondimensional time step of $\Delta t^{+}=\Delta t u_{\tau}^{2} / v=$ 0.1 in wall units. Here, $u_{\tau}$ is the wall friction velocity, and $v$ is the kinematic viscosity.

The instantaneous streamwise velocity fluctuation field is illustrated in Fig. 9, which exhibits the typical streak pattern of the wall-bounded turbulence. The time- and spanwise-averaged velocity profile at $x=6 \delta$ is shown in Fig. 10. The van Driest-transformed velocity profile solved by the low-dissipative solver exhibits a satisfactory agreement with the DNS [36] and accords with the log law. Figure 11 shows the streamwise, spanwise, and wall-normal rms of velocity fluctuations and Reynolds shear-stress profiles at $x=6 \delta$. One can notice that all the velocity fluctuations agree convincingly with the statistical distributions obtained by pure LES [36] and DNS [37]. In general, this case proves that the low-dissipative solver is appropriate in resolving wall-bounded turbulence, and thus it has the potential to deal with complicated wall-bounded turbulent flows.

\section{E. Application to Realistic Flow}

To validate the low-dissipative solver's performance in the modeling of a realistic scramjet, a supersonic flow flushing over a rearward-facing step and subsequently reattached along an inclined wall is investigated. The flow contains massive flow separations as well as shock-wave/turbulent-boundary-layer interactions, which are commonly encountered in hypersonic propulsion flowpath. Thus, a detailed validation against the measurements, especially for the velocity profiles along the shear layer and above the ramp wall, will be conducted to build confidence in the accuracy of the lowdissipative solver in modeling complex supersonic turbulent flows.

The flow chosen for the current investigation has been extensively studied by Baca [38] and Settles et al. [39] in a high-Reynoldsnumber, blowdown, supersonic wind tunnel. Nominal conditions in the experiment tests are set as a stagnation pressure of $p_{0}=$ $0.69 \mathrm{MPa} \pm 1 \%$, a stagnation temperature of $T_{0}=258 \mathrm{~K} \pm 4 \%$, a freestream Mach number of $M_{\infty}=2.92 \pm 0.015$, and a freestream unit Reynolds number of $6.7 \times 10^{7} / \mathrm{m} \pm 4 \%$. Figure 12 schematically shows the basic geometry used in the experiment. The test model consists of a wedge-shaped plate with a 25.4-mm-deep and 61.9-mm-long cavity, followed by a 160-mm-long ramp at an inclination angle of $20 \mathrm{deg}$. To promote the two-dimensionality of the experiment, aerodynamic fences were attached to the ramp and extended above the cavity.

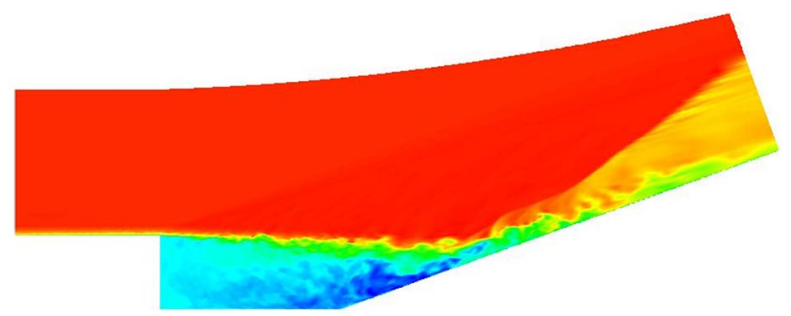

a)

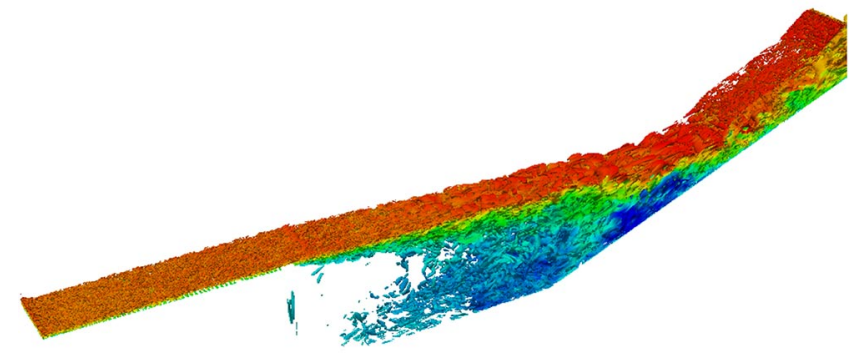

b)

Fig. 15 Instantaneous flow structure of the computational domain: a) streamwise velocity field, and b) turbulent structures visualized via the Q-criterion with mapped-on streamwise velocity.

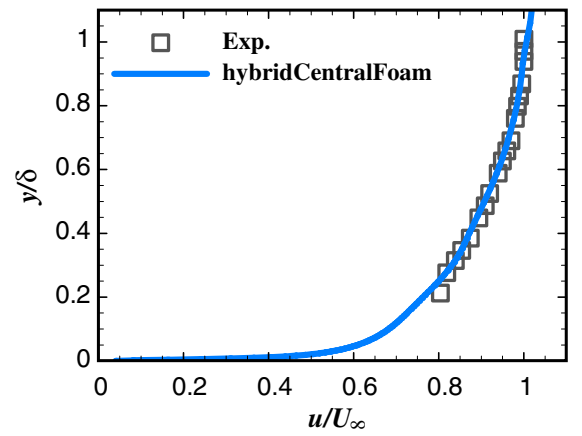

a)

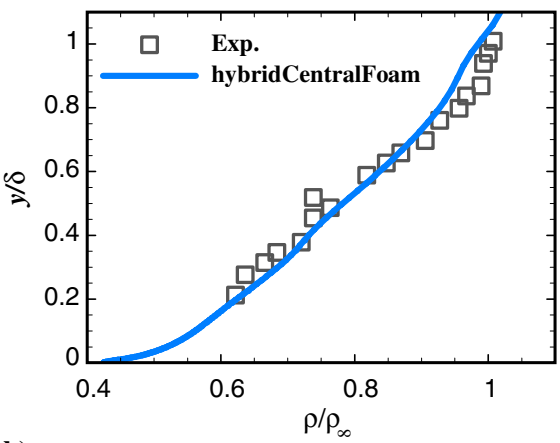

b)

Fig. 14 Comparison of experimentally measured and extracted boundary-layer profiles: a) velocity, b) density, and c) temperature at $25.4 \mathrm{~mm}$ upstream of the cavity.

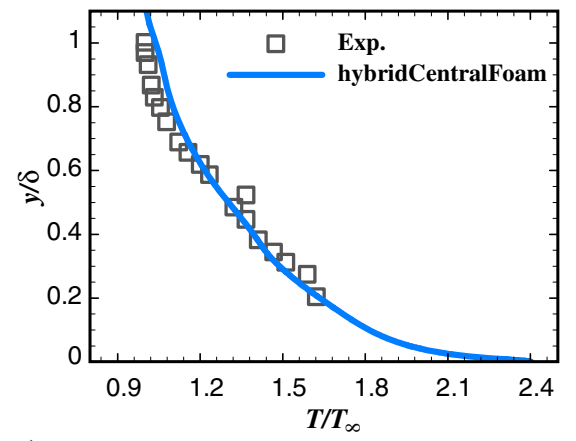

c) 


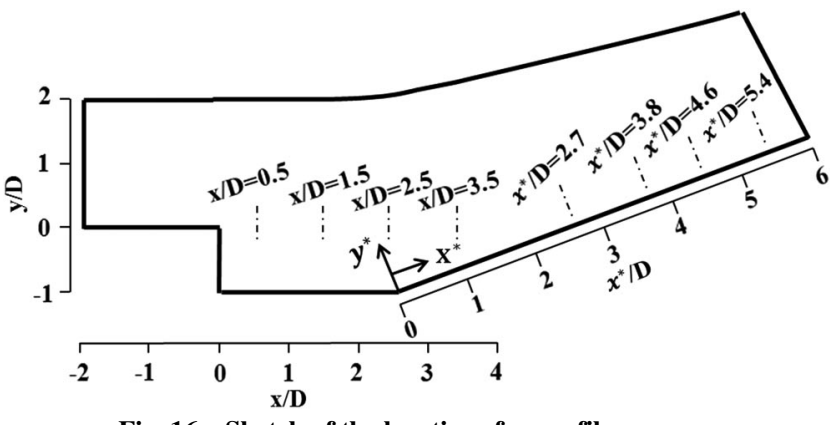

Fig. 16 Sketch of the locations for profile surveys.

Simulations are performed on a three-dimensional grid with approximately 16 million cells, as shown in Fig. 13 . The physical dimensions in the streamwise, spanwise, and wall-normal directions of the computational domain are respectively $26.2,1.3$, and $5.0 \mathrm{~cm}$. The flat plate extended upstream for a distance of two step heights to allow sufficient distance for the inflow turbulence to develop into the fully developed state. Downstream of the backward-facing step, a more isotropic grid is generated for the free shear layer. The grid is clustered to provide a nominal $y^{+} \approx 1.0$ adjacent to the surface. The flowfield is initialized with a converged flowfield simulated by a RANS simulation on a coarser grid. Statistics are taken after the flowfield evolves for a minimum of three characteristic flowthrough times.

Accurate description of the turbulence behaviors in the upstream is a key step in modeling the complex shock/turbulence interaction in the downstream. In the current simulation, adiabatic and no-slip boundary conditions are assumed for all the walls, and periodic boundary conditions are applied in the spanwise direction. To

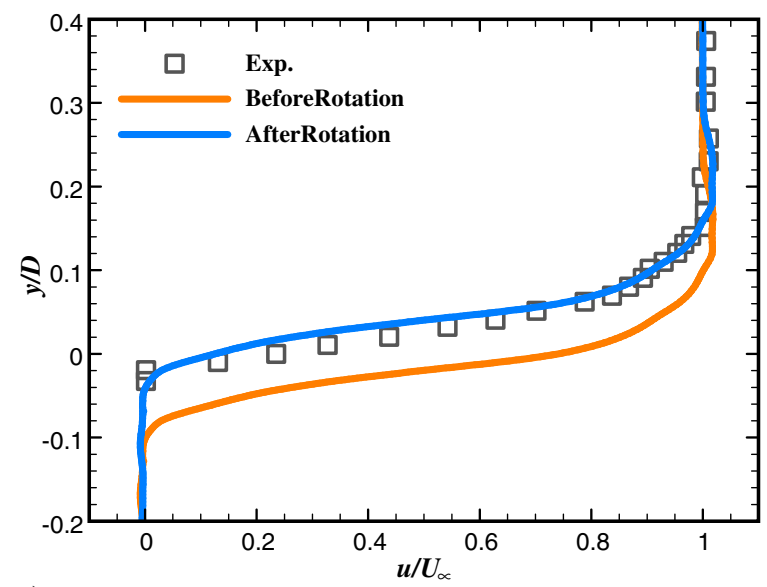

a)

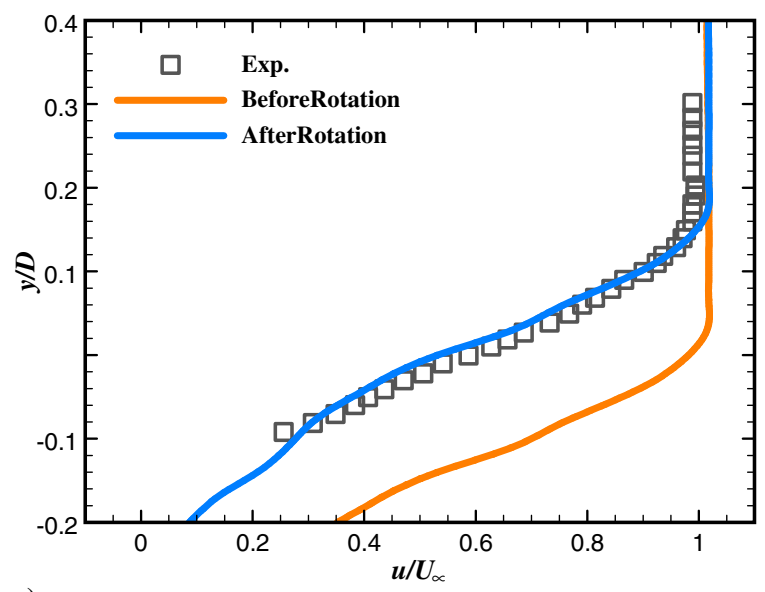

introduce coherent turbulent structures on the inflow boundary, the synthetic turbulence generation method is employed for the velocity and temperature profiles. As a preliminary verification of the synthetic turbulence method, Fig. 14 compares the time-averaged velocity (Fig. 14a), density (Fig. 14 b), and temperature profiles (Fig. 14c) between the experimental measurements and the extracted boundary layer from current modeling at $25.4 \mathrm{~mm}$ upstream of the cavity. The results demonstrate that the synthetic turbulence method can correctly reproduce the mean flow condition upstream of the cavity. The synthetic turbulent inflow will provide massive turbulent fluctuations and rich coherent turbulent structures for the downstream, which are mostly resolved by the current grid setting and IDDES approach.

The instantaneous streamwise velocity contours are highlighted in Fig. 15a. The major flow features observed in the experiment are all well reproduced in the modeling. The turbulent inflow evolves along the flat plate and then detaches at the corner of the backward-facing step, creating a free shear layer over the cavity. When it reattaches along the ramp, the free shear layer will interact with an oblique shock front standing off the ramp, and the flow will be entrained into the cavity near the reattachment point. Figure $15 \mathrm{~b}$ presents the contour image of the turbulent structure visualized via the isosurfaces of Q-criterion with mapped-on streamwise velocity, which shows that fine-scale wall-bounded turbulent structures slowly evolve along the flat plate in the upstream. As the flow detaches from the corner of the backward-facing step, much larger vortex structures come into being in the free shear layer and recirculation zone. The recompression shock after reattachment also has a pronounced effect on the turbulence structure, which merits further studies.

The measurements at different locations denoted in Fig. 16 for the shear layer above the cavity and the boundary layer along the ramp are compared to validate the modeling results. Here, the streamwise

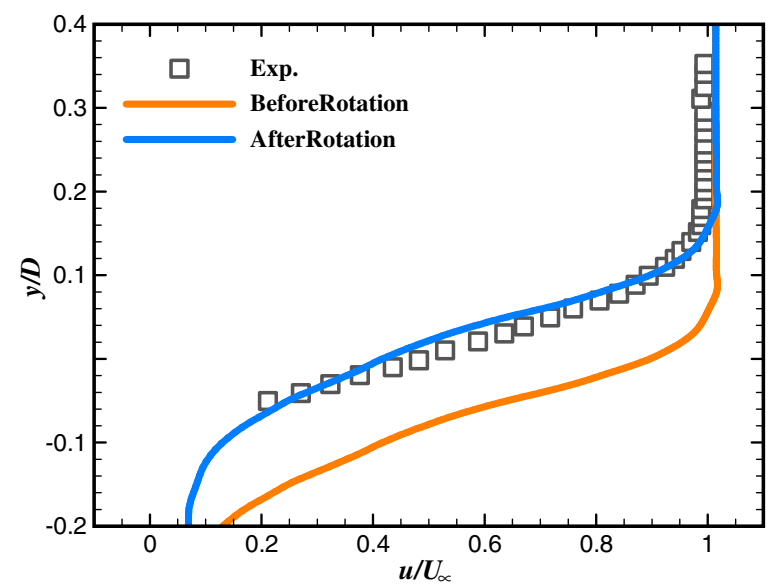

b)

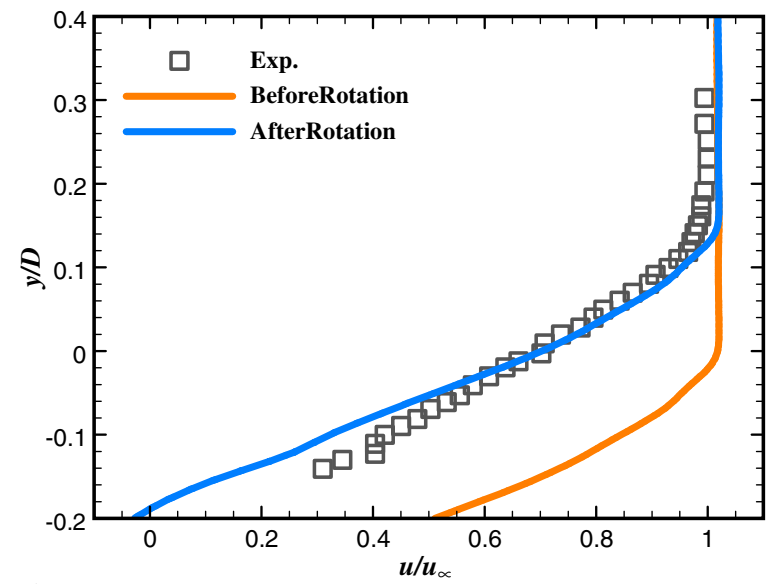

d)

Fig. 17 Normalized velocity profiles through the shear layer: a) $x=0.5 D$, b) $x=1.5 D$, c) $x=2.5 D$, and d) $x=3.5 D$. 


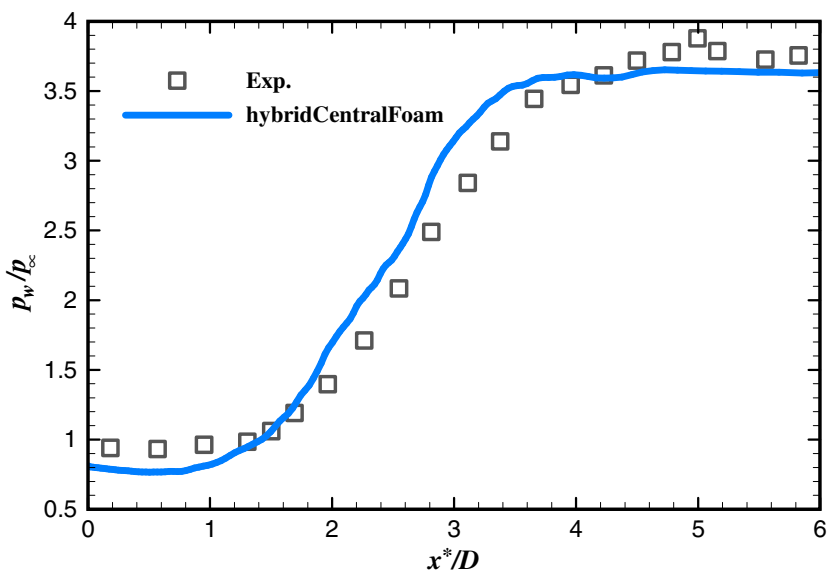

Fig. 18 Normalized wall pressure distribution along the reattachment ramp.

and normal distances have been normalized by the step height of $D=25.4 \mathrm{~mm}$. Comparisons of the time-averaged normalized velocity profiles through the shear layer are presented in Fig. 17. In the modeling, the flowfield is actually treated as a quasi-twodimensional problem because the cyclic boundary conditions are applied in the spanwise direction. However, such treatment may lead to the flow not being totally reproduced. In this case, the lower pressure in the cavity, the downward deflection of the free shear layer, and the slight expansion above the cavity can possibly be attributed to insufficient consideration of the three-dimensionality, which causes a slight inclination of the predicted shear direction [40]. To make a

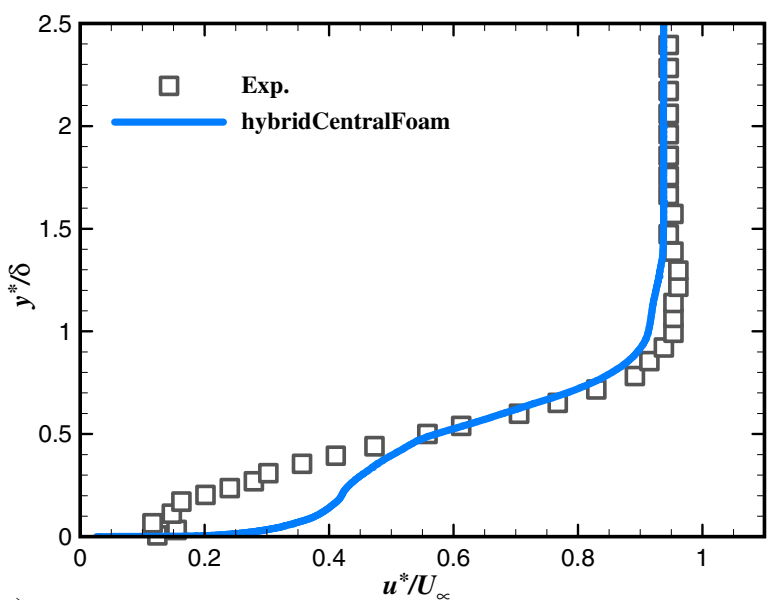

a)

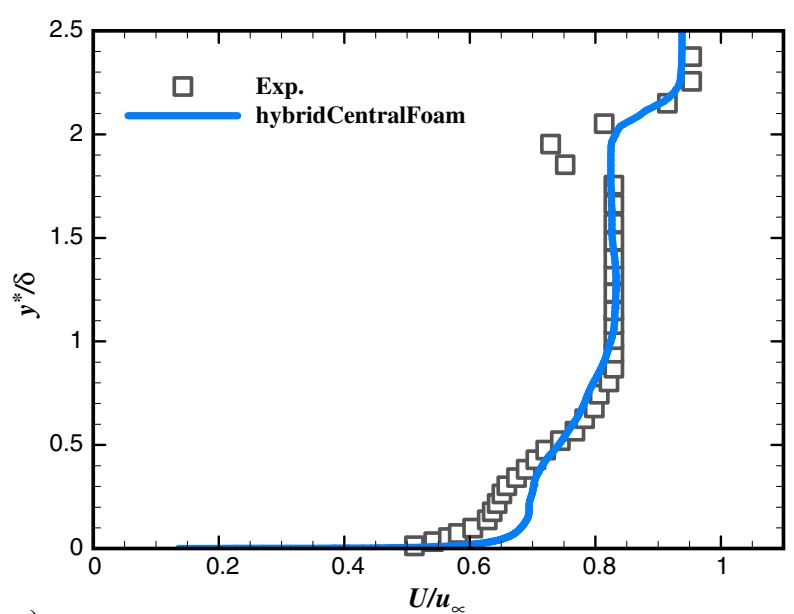

better comparison of the spreading rate of the shear layer, the predicted shear layer is rotated by a small angle to line up with the actual direction of the shear layer in the experiment. After the rotation, the velocity profiles through the shear layer agree quite well with the measured spreading rate, as depicted in Fig. 17.

Comparisons of the normalized static pressure along the ramp wall and velocity profiles across the ramp boundary layer are shown in Figs. 18 and 19, where the predictions are in reasonable agreement with the measurements but still with some discrepancies at some locations. The discrepancies are possibly caused by the lower pressure at the bottom of the ramp due to a slight expansion of the upstream shear layer, and the earlier pressure rising as the downward deflection of the shear layer collides with the ramp. Those also explain the mismatch of the predicted velocity profiles with the measurements in the inner boundary layer on the reattachment ramp in Fig. 19. As the free shear layer deflects downward, the downstream flowfield will experience an earlier occurrence of flow reattachment. Thereby, the inner velocity profiles along the ramp in the downstream will undergo premature boundary-layer recovery state, though the velocity profiles in the outer boundary layer agree well with the experimental data.

\section{Conclusions}

In this study, a low-dissipative hybrid compressible solver for supersonic turbulent flows is developed within the unstructured OpenFOAM framework. The new solver adopts a hybridized scheme, combining a dissipative scheme with excellent shockcapturing capability and the central scheme with nondissipative property, for the purpose of accurate modeling of both shock and turbulence in the supersonic turbulent flows. The shock sensor plays

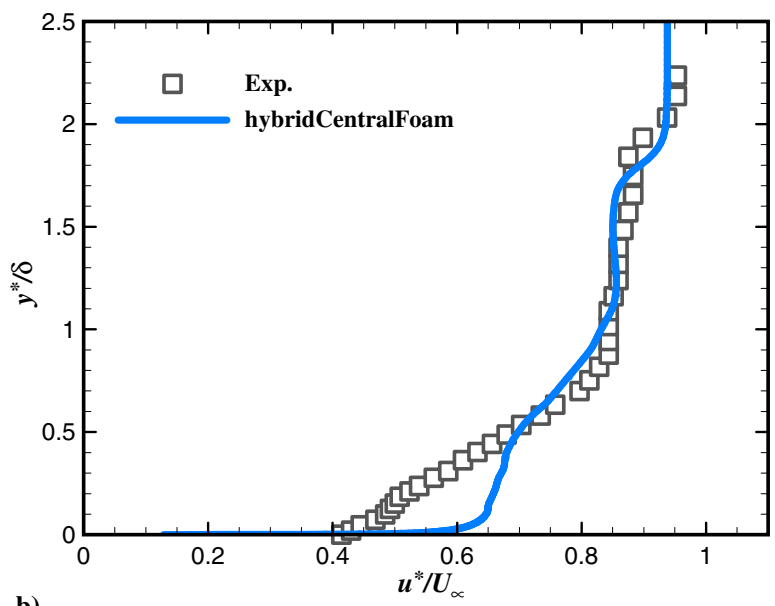

b)

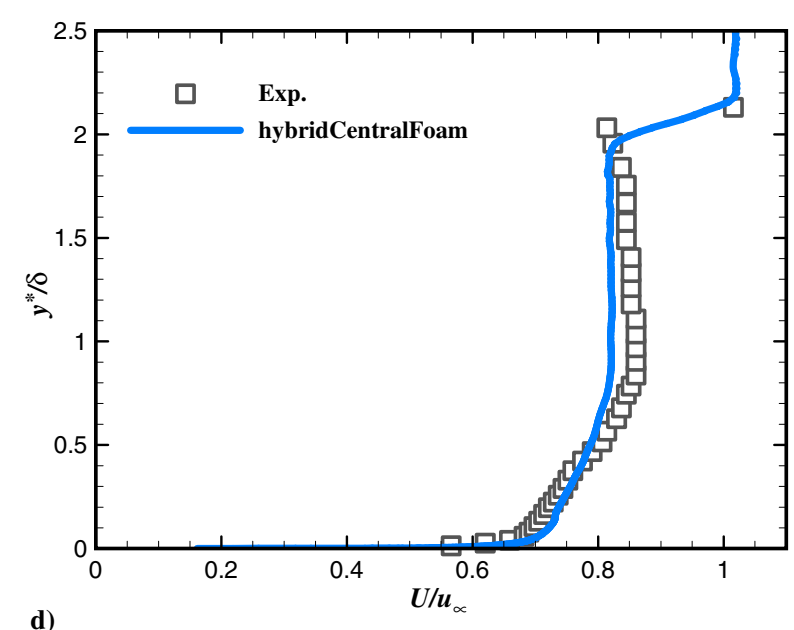

d)

Fig. 19 Normalized velocity profiles normal to the reattachment ramp surface: a) $x^{*}=2.7 D$, b) $x^{*}=5.4 D$. 
a key role in the hybridization formulation and determines the numerical dissipation of the flow modeling to a large degree. Here, a modified Ducros sensor is employed to activate the dissipative scheme only in the vicinity of strong shock waves. Furthermore, the third-order total variation diminishing Runge-Kutta time-marching method and a hybrid RANS/LES approach based on the $k-\omega$ SST model is implemented in the low-dissipative hybrid solver to reduce the temporal dissipation and to deal with wall-bounded turbulent flows.

The hybrid-scheme-based solver was validated by a series of benchmark problems, including the Sod shock-tube test, the ShuOsher problem, the Green-Taylor vortex evolution, and a wallbounded turbulent flow. The performance of the low-dissipative solver in both resolving turbulence and capturing flow discontinuities was validated through extensive comparisons with the experimental data and the DNS results. In addition, the synthetic turbulence method coupled with the low-dissipative solver proved to be an effective method in generating compressible turbulence inflow for LES. The modeling of supersonic flow over a cavity ramp configuration further validated the low-dissipative solver's reliability and robustness in resolving the flowfield with both flow discontinuities and multiple-scale turbulence. At the same time, the test case also emphasizes the importance of three-dimensional effects in modeling realistic flows.

\section{Acknowledgments}

The project was supported by the National Natural Science Foundation of China (grant 11502270) and the Training Program of the Major Research Plan of the National Natural Science Foundation of China (grant 91641110). The authors are also grateful to the National Supercomputer Center in Tianjin for providing the computational resource.

\section{References}

[1] Jameson, A., Schmidt, W., and Turkel, E., "Numerical Solution of the Euler Equations by Finite Volume Methods Using Runge-Kutta Time Stepping Schemes," 14th Fluid and Plasma Dynamics Conference, AIAA Paper 1981-1259, 1981.

doi:10.2514/6.1981-1259

[2] von Neumann, J., and Richtmyer, R. D., "A Method for the Calculation of Hydro-Dynamical Shocks," Journal of Applied Physics, Vol. 21, No. 3, 1950, pp. 232-237.

doi:10.1063/1.1699639

[3] Pirozzoli, S., "Numerical Methods for High-Speed Flows," Annual Review of Fluid Mechanics, Vol. 43, 2011, pp. 163-194. doi:10.1146/annurev-fluid-122109-160718

[4] Phillips, A., "An Example of Nonlinear Computational Instability," In the Atmosphere and the Sea in Motion, Oxford Univ. Press, Oxford, England, U.K., 1959, pp. 501-504.

[5] Honein, A., and Moin, P., "Higher Entropy Conservation and Numerical Stability of Compressible Turbulence Simulations," Journal of Computational Physics, Vol. 201, No. 2, 2004, pp. 531-545. doi:10.1016/j.jcp.2004.06.006

[6] Peterson, D. M., "Simulation of Injection, Mixing, and Combustion in Supersonic Flow Using a Hybrid RANS/LES Approach," Ph.D. Dissertation, Univ. of Minnesota, Minneapolis, MN, 2011.

[7] Potturi, A., and Edwards, J. R., "Hybrid Large-Eddy/ReynoldsAveraged Navier-Stokes Simulations of Flow Through a Model Scramjet," AIAA Journal, Vol. 52, No. 7, 2014, pp. 1417-1429. doi:10.2514/1.J052595

[8] Modesti, D., and Pirozzoli, S., "A Low-Dissipative Solver for Turbulent Compressible Flows on Unstructured Meshes, with OpenFOAM Implementation," Computers \& Fluids, Vol. 152, April 2017, pp. 14-23. doi:10.1016/j.compfluid.2017.04.012

[9] Adams, N. A., and Shariff, K., "A High-Resolution Hybrid CompactENO Scheme for Shock-Turbulence Interaction Problems," Journal of Computational Physics, Vol. 127, No. 1, 1996, pp. 27-51. doi:10.1006/jcph.1996.0156

[10] Hill, D., Pantano, C., and Pullin, D., "Large-Eddy Simulation and Multiscale Modeling of a Richtmyer-Meshkov Instability with Reshock," Journal of Fluid Mechanics, Vol. 557, June 2006, pp. 29-61. doi: $10.1017 / \mathrm{S} 0022112006009475$
[11] Pantano, C., Deiterding, R., Hill, J., and Pullin, D., "A Low Numerical Dissipation Patch-Based Adaptive Mesh Refinement Method for Large-Eddy Simulation," Journal of Computational Physics, Vol. 221, No. 1, 2007, pp. 63-87. doi:10.1016/j.jcp.2006.06.011

[12] Ducros, F., Ferrand, V., Nicoud, F., Wber, C., Darracq, D., Gacherieu, C., and Poinsot, T., "Large Eddy-Simulation of the Shock/Turbulence Interaction," Journal of Computational Physics, Vol. 152, No. 2, Feb. 1999, pp. 517-549.

doi:10.1006/jcph.1999.6238

[13] Lombardini, M., "Richtmyer-Meshkov Instability in Converging Geometries," Ph.D. Dissertation, Cailifornia Inst. of California, Pasadena, CA, 2008.

[14] Pirozzoli, S., "Numerical Methods for High-Speed Flows," Аnnиal Review of Fluid Mechanics, Vol. 43, 2011, pp. 163-194. doi:10.1146/annurev-fluid-122109-160718

[15] Khalighi, Y., Nichols, J. W., and Ham, F., "Unstructured Large Eddy Simulation for Prediction of Noise Issued from Turbulent Jets in Various Configurations," 17th AIAA/CEAS Aeroacoustics Conference, AIAA Paper 2011-2886, June 2010. doi:10.2514/6.2011-2886

[16] Vuorinen, V., Larmi, M., Schlatter, P., Fuchs, L., and Boersma, B. J., “A Low-Dissipative Scale-Selective Discretization Scheme for the Navier-Stokes Equations," Computers \& Fluids, Vol. 70, Nov. 2012, pp. 195-205. doi:10.1016/j.compfluid.2012.09.022

[17] Kurganov, A., and Tadmor, E., "New High-Resolution Central Schemes for Nonlinear Conservation Laws and Convection-Dissipation Equations," Journal of Computational Physics, Vol. 160, No. 1, 2000, pp. 241-282. doi: $10.1006 /$ jcph.2000.6459

[18] Lax, P., "Weak Solutions of Nonlinear Hyperbolic Equations and Their Numerical Computation," Communications on Pure and Applied Mathematics, Vol. 7, No. 1, 1954, pp. 159-193. doi: 10.1002/(ISSN)1097-0312

[19] Nessyahu, H., and Tadmor, E., "Non-Oscillatory Central Differencing for Hyperbolic Conservation Laws," Journal of Computational Physics, Vol. 87, No. 2, 1990, pp. 408-463. doi:10.1016/0021-9991(90)90260-8

[20] Greenshields, C., Weller, H., Gasparini, L., and Reese, J., "Implementation of Semi-Discrete, Non-Staggered Central Schemes in a Colocated, Polyhedral, Finite Volume Framework, for High-Speed Viscous Flows," International Journal for Numerical Methods in Fluid, Vol. 63, No. 1, 2010, pp. 1-21. doi:10.1002/fld.2069

[21] Feiereisen, J., Reynolds, C., and Ferziger, H., "Numerical Simulation of a Compressible, Homogeneous, Turbulent Shear Flow," NASA CR164953, 1981.

[22] Kennedy, C. A., and Gruber, A., "Reduced Aliasing Formulations of the Convective Terms Within the Navier-Stokes Equations," Journal of Computational Physics, Vol. 227, No. 3, 2008, pp. 1676-1700. doi:10.1016/j.jcp.2007.09.020

[23] Pirozzoli, S., "Generalized Conservative Approximations of Split Convective Derivative Operators," Journal of Computational Physics, Vol. 229, No. 19, 2010, pp. 7180-7190. doi:10.1016/j.jpp.2010.06.006

[24] Morris, C., "Studies of Inviscid Flux Schemes for Acoustics and Turbulence Problems," 51 st AIAA Aerospace Sciences Meeting, AIAA Paper 2013-0075, Jan. 2013.

[25] Bhagatwala, A., and Lele, S., "A Modified Artificial Viscosity Approach for Compressible Turbulence Simulations," Journal of Computational Physics, Vol. 228, No. 14, April 2009, pp. 4965-4969. doi:10.1016/j.jcp.2009.04.009

[26] Shu, C. W., and Osher, S., "Efficient Implementation of Essentially Non-Oscillatory Shock Capturing Schemes," Journal of Computational Physics, Vol. 77, No. 2, 1988, pp. 439-471. doi:10.1016/0021-9991(88)90177-5

[27] Gottlieb, S., and Shu, C. W., "Total Variation Diminishing Runge-Kutta Schemes," Mathematics of Computation, Vol. 67, No. 221, 1998, pp. $73-85$. doi:10.1090/mcom/1998-67-221

[28] Gottlieb, S., Shu, C. W., and Tadmor, E., "Strong Stability-Preserving High-Order Time Discretization Methods," SIAM Review, Vol. 43, No. 1, 2001, pp. 89-112. doi:10.1137/S003614450036757X

[29] Gritskevich, M. S., Garbaruk, A. V., Schutze, J., and Menter, F. R., "Development of DDES and IDDES Formulations for the $k-\omega$ Shear Stress Transport Model," Flow, Turbulence and Combustion, Vol. 88, 
No. 3, 2012, pp. 431-449.

doi:10.1007/s10494-011-9378-4

[30] Sod, G. A., "A Survey of Several Finite Difference Methods for Systems of Nonlinear Hyperbolic Conservation Laws," Journal of Computational Physics, Vol. 27, No. 1, 1978, pp. 1-31. doi:10.1016/0021-9991(78)90023-2

[31] Shu, C., and Osher, S., "Efficient Implementation of Essentially NonOscillatory Shock-Capturing Schemes," Journal of Computational Physics, Vol. 83, No. 1, 1989, pp. 32-78.

doi:10.1016/0021-9991(89)90222-2

[32] Johnsen, E., Larsson, J., Bhagatwala, A. V., Cabot, W. H., Moin, P., Olson, B. J., Rawar, P. S., Shankar, S. K., Sjogreen, B., Yee, H. C., Zhong, X., and Lele, S. K., "Assessment of High-Resolution Methods for Numerical Simulations of Compressible Turbulence with Shock Waves," Journal of Computational Physics, Vol. 229, No. 4, 2010, pp. 1213-1237. doi:10.1016/j.jcp.2009.10.028

[33] Duponcheel, M., Orlandi, P., and Winckelmans, G., "Time-Reversibility of the Euler Equations as a Benchmark for Energy Conserving Schemes," Journal of Computational Physics, Vol. 227, No. 19, 2008, pp. 8736-8752. doi:10.1016/j.jcp.2008.06.020

[34] Brachet, M. E., Meiron, D. I., Orszag, S. A., Nickel, B. G., Morf, R. H., and Frisch, U., "Small-Scale Structure of the Taylor-Green Vortex," Journal of Fluid Mechanics, Vol. 130, May 1983, pp. 411-452. doi:10.1017/S0022112083001159

[35] Hillewaert, K., "Testcase Description C3.5: Direct Numerical Simulation of the Taylor-Green Vortex at $R e=1600, " 1 s t$
International Workshop on High-Order CFD Methods at the 50th AIAA Aerospace Sciences Meeting, AIAA, Reston, VA, 2012, http://dept.ku. edu/ cfdku/hiocfd.html [retrieved Oct. 2016].

[36] Roidl, B., Meinke, M., and Schroder, W., "A Reformulated Synthetic Turbulence Generation Method for a Zonal RANS-LES Method and its Application to Zero-Pressure Gradient Boundary Layers," International Journal of Heat and Fluid Flow, Vol. 44, No. 3, 2013, pp. $28-40$ doi:10.1016/j.ijheatfluidflow.2013.03.017

[37] Pirozzoli, S., and Bernardini, M., "Turbulence in Supersonic Boundary Layers at Moderate Reynolds Number," Journal of Mechanics, Vol. 688, Dec. 2011, pp. 120-168. doi: $10.1017 / \mathrm{jfm} .2011 .368$

[38] Baca, B. K., "An Experimental Study of the Reattachment of a Free Shear Layer in Compressible Turbulent Flow," M.S. Thesis, Princeton Univ., Princeton, NJ, 1981.

[39] Settles, G. S., Williams, D. R., Baca, B. K., and Bogdonoff, S. M., "Reattachment of a Compressible Turbulent Free Shear Layer," AIAA Journal, Vol. 20, No. 1, 1982, pp. 60-67. doi: $10.2514 / 3.51047$

[40] Leger, T., Bisek, N., and Poggie, J., "Detached-Eddy Simulation of a Supersonic Reattaching Shear Layer," AIAA Journal, Vol. 55, No. 11, 2017, pp. 3722-3733. doi:10.2514/1.J056103

H. Blackburn Associate Editor 